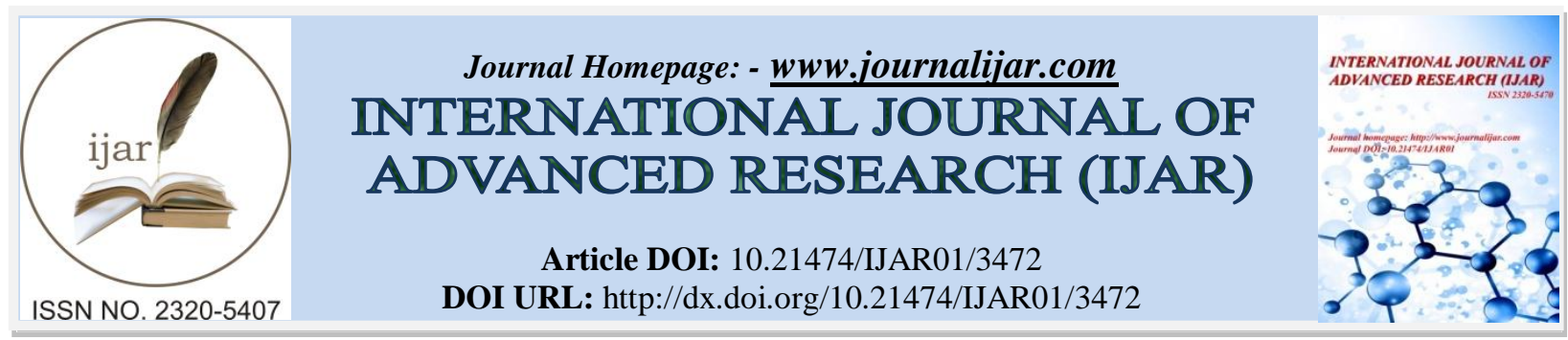

RESEARCH ARTICLE

\title{
ANALYSIS OF CHANGES IN BILATERAL MASSETER AND ANTERIOR TEMPORALIS MUSCLE EFFICIENCY IN COMPLETE DENTURE WEARERS. -AN EMG STUDY.
}

\section{Dr. Shilpa Sinnurkar ${ }^{1}$, Dr. Saquib Ahmed Shakh ${ }^{2}$ and Dr. Ramesh Nadiger ${ }^{3}$.}

1. Assistant Professor, Department Of Prosthodontics, Coorg Institute Of Dental Sciences Virajpet -571218 Karnataka India.

2. Associate Professor, Department Of Prosthodontics, Sdm College Of Dental Sciences Sattur, Dharwad Karnataka India.

3. Professor And Hod, Department Of Prosthodontics, Sdm College Of Dental Sciences Sattur, Dharwad Karnataka India.

\section{Manuscript Info}

Manuscript History

Received: 22 December 2016

Final Accepted: 20 January 2017

Published: February 2017

Key words:-

Electromyography, masticatory muscles efficiency, motor units, complete denture, maximum voluntary contraction.

\begin{abstract}
Purpose: The loss of natural teeth leads to bone resorption and muscular hypotonicity, which may affect the structures involved in mastication. Atrophy of supporting tissues, poor adaptation, reduced masticatory efficiency, and psychosocial embarrassment are major complaints of edentulous patient wearing old/faulty denture. The need for this study is to examine the effect of old/faulty dentures and a new denture on the masticatory muscle efficiency with the help of electromyography.

Materials and methods: 15 edentulous patients wearing complete denture prosthesis selected as a subject for the investigation. Surface electrodes from electromyographic unit were placed in the region of right and left anterior Temporalis muscle and Masseter muscle, and the patients will be asked to perform maximum voluntary contraction. The muscle activity analyzed twice for each patient: (1) with the old/faulty dentures and (2) 5 month following insertion of new denture.

Results: The electrical activity during maximum voluntary contraction exhibited statistically significant improved muscle efficiency with new denture when compared to those with old dentures in place for 5 months.

Conclusion: New dentures or improvements in occlusion and vertical dimension produce a positive benefit to the patient by reducing the muscle effort during chewing without affecting masticatory performances. The reduction in muscle effort is likely to cause less tissue and in the end may minimize residual ridge resorption and new complete denture allows for neuromuscular reprogramming, which contributes to muscular balance of the masticatory system.
\end{abstract}

Copy Right, IJAR, 2017,. All rights reserved.

\section{Introduction:-}

The "stomatognathic system" is complex system that acts as a functional unit of the body characterized by several tissues of different origin and structure that act in consonance to perform different functional tasks. It comprises the 
joint connection between the mandible and the base of the skull, which in turn has muscle and ligament connections to the neck region. It consists of skeletal components (maxilla and mandible), dental arches, soft tissue (salivary glands, nervous and vascular supplies) temporomandibular joints (TMJ) and muscles. These structures are interconnected and interrelated; when activated, they aim to achieve maximal efficiency with protection of all the participating tissues. The masticatory system is also a part of this complex stomatognathic system. In dentulous subjects, mastication is highly coordinated neuromuscular function that involves fast effective movements of the jaw and continuous modulation of forces in craniofacial system. Masticatory functions generated by rhythmic contraction of masticatory muscles. These muscles have capacity to contract during daily use of the stomatognathic system and maintain the muscle tone in mandibular rest position. Whereas, in edentulous subjects these mechanism acts with marked differences. The loss of natural teeth leads to bone resorption and muscular hypotonicity, which may affect the structures involved in mastication leading to reduced capacity in various functions of stomatognathic system[1].

When teeth are lost, a change occurs in the mandibular posture, the speech pattern, esthetics, and deglutition as well as in the individual's social, emotional and psychological behavior. Along with this, some age related changes, such as deterioration in the fast and slow-twitch fibers of the striated muscles, results in impaired muscle force. Edentulous persons have reduced capacity to perform various functions of the stomatognathic system such as occlusal forces, tactile thresholds and chewing ability. The loss of teeth and elimination of periodontal afferent innervations flow lead to changes in the neuromuscular patterns causing the reduced chewing efficiency when new complete denture replaces teeth[2]. For the patient who has previously worn dentures, the clinician must determine the OVD(occlusion at vertical dimension) based on the clinical presentation. By establishing, an appropriate OVD in the patient who has worn the same dentures for 15 years or longer can be challenging. Excessive wear of the denture teeth in a long-term wearer can cause the patient to function at a reduced OVD, possibly compromising the oral cranio-facial system. In addition, older denture wearers may suffer age-related morphologic changes, such as decreased facial height, because of mandibular ridge resorption and a downward and forward rotation of the mandible[1]. An increase in the vertical dimension may lead to changes in the oro-facial structures, i.e. jaw elevator muscles and temporo-mandibular joints. An increase in the vertical dimension may influence the masticatory performance both in a negative way, (hampered chewing cycle) and in a positive way (increased bite force).

Although, various techniques are available for examining the stomatognathic system, the electromyographic recording is one of the convenient and useful methods because it directly measures muscle activity. Electromyographic techniques have permitted more precise assessment of the muscle functions compared to previously possible clinical observation. Technological development has led to the optimization and application of computerized diagnostic systems such as electro-gnatho-graphy[3]. The first effort to apply electromyography in dentistry was made by Robert E. Moyers. It used to corroborate the neuropsychological analysis of the factors linked to prosthetic rehabilitation procedures. Electromyographic data representing muscle activity during movements of the mandible (e.g.chewing) are the result of several variables which are operating simultaneously-i.e., continual variation in muscle length, bite dimension, and temporomandibular joint axis. The study of such data has contributed to our knowledge of the relative function of the masticatory muscles and has certainly indicated the potential value of electromyographic data in dentistry[4].

\section{Materials and Methods:-}

\section{Sample selection:-}

Fifteen edentulous patients (eight men and seven women aged between 40 and 80 years) with complete, old maxillary and mandibular dentures, worn for over 10 years were selected for the present study. The patients were selected from the out patient department of Prosthodontics. All patients presented diminished occlusal vertical dimension (OVD), with deficient dental occlusion, and severe bone resorption, particularly of the mandibular arch. They were all asymptomatic and presented no signs or symptoms of neuromuscular and temporomandibular disorders (TMD). The selected patients were informed about the treatment to be instituted and signed a term of consent in accordance with the recommendations of the Human Research Ethics Committee.

\section{Screening examination:-}

The computerized system was used for recording the EMG data. The components of this system comprises of a display monitor, a cathode ray tube and electrodes (surface and hypodermic needle). The monitor helps in screening and it display the amplitude of muscle activity by special RMS EMG software (figure no 1). The cathode ray tube (CRT) transmits the activity of muscle from electrode to the monitor, and the electrode, which filters the muscle 
activity and it, transmits to the CRT for amplification. Surface EMG utilizes sensing electrodes placed on the skin, which allows the clinician to directly and accurately monitor muscle activity.

\section{Preparation of the patients:-}

On the day of EMG recording for old denture and with the new denture after 5-months follow up, any needed adjustment to the intaglio, cameo and occlusal surfaces of the complete dentures were made. During the electromyographic recording (figure no. 1), the patient was asked to seat on nonconductive chair, which reduces the interferences in an upright but relaxed position. The head was kept unsupported, and aligned according to Frankfort horizontal plane, parallel to the floor. The conductivity of the electrode-skin interface was increased using conductive gel after thorough cleaning of the skin with 99.5\% alcohol swabs and skin preparation gel to enable an easy transport of electrical potentials. The electrode must be firmly attached to the skin, with adhesive tape, to avoid bias due to movements (figure no 2). The application of conductive gel reduces the impedance below $20 \mathrm{kV}$. The recordings were performed 5 minutes later, which allowed the conductive paste to adequately moisten the surface. The EMG signals were filtered (0.003-1.0) and amplified with a time constant of 10mili second, and displayed on the cathod ray tube (CRT) monitor for on line monitoring.

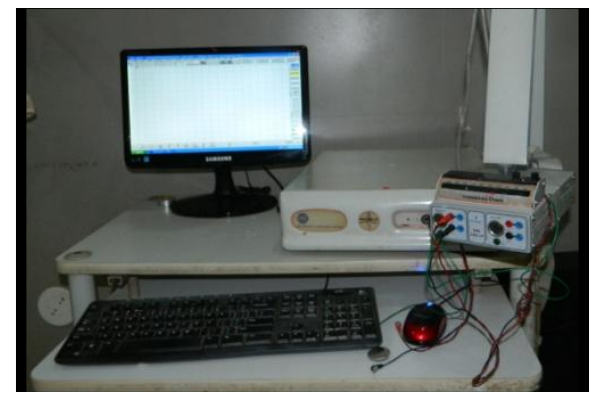

Figure No1:- Electromyogram.

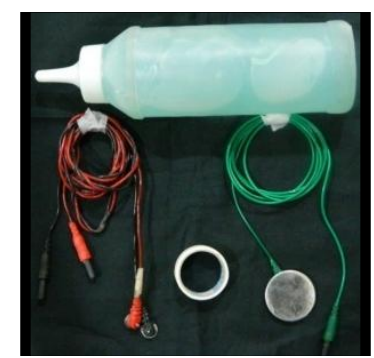

Figure No 2:- Electrodes, Conductive Gel \& Adhesive Tape.

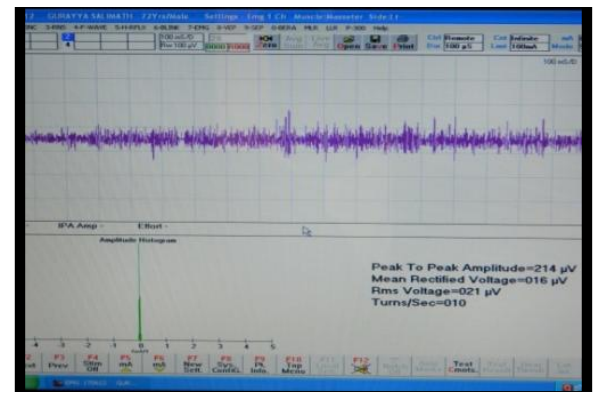

Figure No 3:- Electromyographic Recording

\section{Experimental protocol:-}

The wires were connected. The appliance is equipped with a trial test, which is necessary to verify the correct state of the electrodes and connections. The anatomical locations for area of interest were recognized and palpated. Each EMG recording lasted $10 \mathrm{~s}$ and a computer calculated the root-mean- square (RMS) value of the EMG activity (figure no.3). The subjects were allowed to practice prior to the start of the experiment. Once the patient and instruments were prepared for the collection of data, the procedure for recording muscle activity by EMG was 
carried out. The electrical signals were captured and the electrical activity of the muscles was analyzed. Each EMG recording was repeated at about a 1-min interval to avoid muscle fatigue. For EMG recording the patient was asked to clench his/her teeth, and keep them clenched for two seconds. Next, the patient was asked to relax the muscles, slightly separating the teeth for another two seconds. The operator controlled all the times. The electrical activity was recorded manually for $5 \mathrm{sec}$, rested for $15 \mathrm{sec}$ and repeated the clenching cycle 5 times. To record maximum tooth clenching, the patient maintained tooth contact for two seconds, and electrical activity was recorded. The averages RMS-EMG of the two repeated recordings were used for the statistical analysis.

\section{Data Collection:-}

EMG recording for bilateral anterior Temporalis muscle: (figure no. 4 and 5):-

For recording EMG activity of anterior Temporalis muscle, two pair of miniature surface bipolar $\mathrm{Ag} / \mathrm{AgCl}$ electrodes were placed over the anterior Temporalis muscle 1-inch posterior and 1-inch superior to the outer canthus of the eye with adhesive tape. These electrodes were placed parallel to the muscle fibers, with a centre-to-centre distance of $30 \mathrm{~mm}$ to avoid the endplate region and thus to obtain stable recordings. They were positioned at the motor point, on each portion of the anterior Temporalis muscles, so that their electrical activity could be assessed. The placement of the electrode was determined by manual palpation of the muscles bilaterally. The muscle activity is checked by asking the patient to gently clench the mouth in maximal intercuspal position following the longitudinal alignment, parallel with the direction of the muscle fibres. The ground electrodes were placed over the center of forehead in order to eliminate possible external interferences. The subjects clenched manually for $5 \mathrm{sec}$ and rested for $15 \mathrm{sec}$ and repeat the clenching cycle 5 times. The highest EMG activity was considered the maximum clenching.

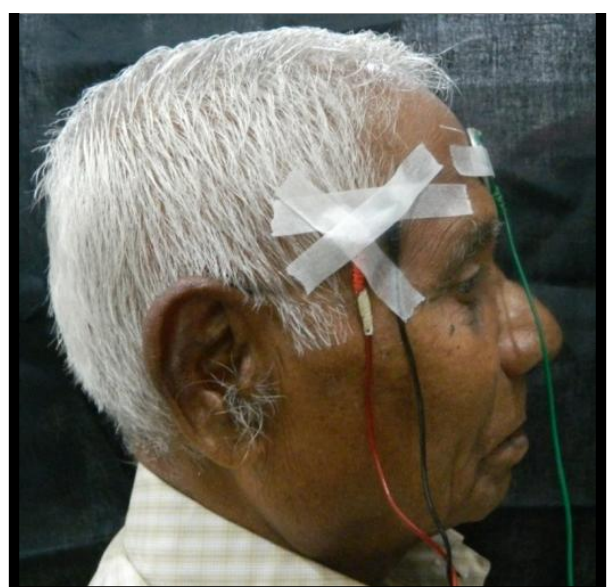

Figure no 4:- Emg Recording For Right Anterior Temporalis Muscles.

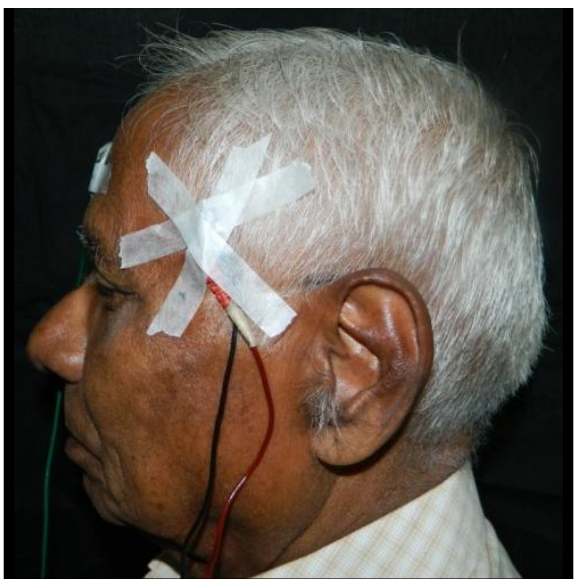

Figure No 5:- Emg Recording For Left Anterior Temporalismuscles 
EMG recording for bilateral Masseter muscle: (figure no. 6 and 7):-

The EMG activity of Masseter muscle was carried out by placing two pair of miniature surface bipolar $\mathrm{Ag} / \mathrm{AgCl}$ electrodes over superficial fibres of Masseter muscle. The superficial fibres of Masseter muscle originate at dorsal surface of zygomatic bone and inserted to the ramus of the mandible. A band of superficial Masseter muscle was palpated by manual method from the prominence of zygoma until the gonial angle of the mandible. The activity this muscle was assessed by forcing the teeth for clenching. These electrodes were placed $2 \mathrm{~cm}$ above the lower margins of the mandible halfway between the mandibular angle and anterior borders of muscles. These electrodes were placed parallel to the Masseter muscle fibers, with a centre-to-centre distance of 30mm to avoid the endplate region and thus to obtain stable recordings. They were positioned at the motor point, on each portion of the muscles, so that their electrical activity could be assessed. The subjects clenched manually for $5 \mathrm{sec}$ and rested for $15 \mathrm{sec}$ and repeat the clenching cycle 5 times. The highest EMG activity was considered the maximum clenching.

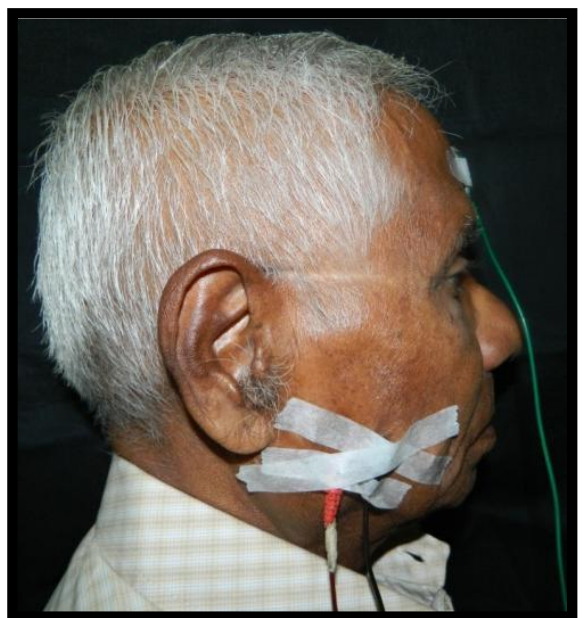

Figure no 6:- Emg Recording For Right Masseterr Muscle.

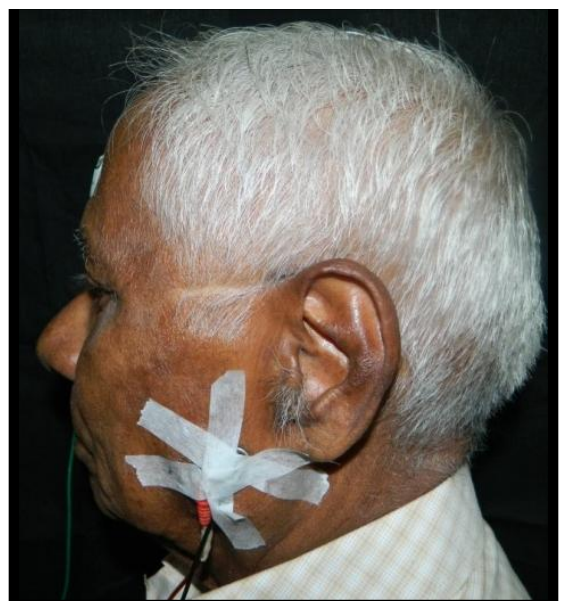

Figure no 7:- Emg Recording For Left Masseterr Muscle.

In all the procedures, the capture and analyses of EMG signals were carried out as recommended by the International Society of Electrophysiology and Kinesiology (Willians 1987). Recordings and analysis of the muscles electrical activity were obtained with the mandible in the maximum voluntary clenching. The EMG signals were stored and analyzed as root mean-square (RMS) values expressed in microvolts $(\mu \mathrm{V})$ in the maximum intercuspation position (figure no. 3). Finally, a new set of complete dentures with corrected occlusal vertical dimensions, an appreciable occlusal anatomy and well-polished cameo surface were delivered to all old denture wearers. The same procedures were performed after 5-months with new set of complete dentures. The statistical analyses were performed using paired t-test. A significance level of 0.05 was adopted for all tests. 


\section{Results:-}

The data engraved from this study were subjected to statistical analyses by using paired t-test. A significance level of 0.05 was adopted for all tests. The data of the study was analyzed and the results are presented in the table 1 and 2. These tables represents the mean values of electromyographic activity of Masseter and anterior Temporalis muscles with old and new denture after 5-months of follow up during maximal voluntary contraction(MVC) in the intercuspal position.

\section{Table 1:-}

This data showed during maximum voluntary contraction of Anterior Temporalis Muscle. The Mean activity of $242.4 \mu \mathrm{V}$ on right side and $237.67 \mu \mathrm{V}$ on left side with old denture and, $168 \mu \mathrm{V}$ on right side and $166.07 \mu \mathrm{V}$ on left side with new denture after 5-months follow up. The paired difference between the old denture and new denture, the activity of Anterior temporalis muscle for right side is 74.4and on left side 71.6, thus from these values it infers that right side of Anterior Temporalis muscle is more efficient than the left side of the muscle. Statistical analysis reveals highly significant increase in muscle efficiency $\left(\mathrm{p}=0.000^{*}, \mathrm{p}=0.000^{*}\right)$ with new denture after 5-months follow up when compared to subjects with old denture during maximum voluntary contraction.

\begin{tabular}{|c|c|c|c|c|c|c|c|}
\hline \multirow{2}{*}{$\begin{array}{c}\text { Anterior } \\
\text { Temporalis Muscle }\end{array}$} & \multicolumn{2}{|c|}{ With old denture $(\mu \mathrm{V})$} & \multicolumn{2}{|c|}{$\begin{array}{c}\text { With new denture } \\
(\mu \mathrm{V})\end{array}$} & \multicolumn{3}{c|}{ Difference Value } \\
\cline { 2 - 8 } & Mean & SD & Mean & SD & Mean & SD & \\
\hline Right & 242.4 & 38.13 & 168 & 32.34 & 74.4 & 42.3 & $0.000^{*}$ \\
\hline Left & 237.67 & 43.26 & 166.07 & 44.15 & 71.6 & 39.9 & $0.000^{*}$ \\
\hline
\end{tabular}

*Significant at $\mathbf{p} \leq \mathbf{0 . 0 5}$

Table 2:- This data showed during maximum voluntary contraction of MasseterMuscle. The mean activity of $231.93 \mu \mathrm{V}$ on right side and $230.60 \mu \mathrm{V}$ on left side with old dentures and, $163.13 \mu \mathrm{V}$ on right side and $171.73 \mu \mathrm{V}$ on left side with new denture after 5-months follow up. paired difference between the old denture and new denture, activity of Masseter muscle for right side is 68.8and on left side 58.8, thus from these values it infers that right side of Masseter muscle is more efficient than the left side of the muscle. Statistical analysis reveals highly significant increase in muscle efficiency $\left(\mathrm{p}=0.000^{*}, \mathrm{p}=0.002^{*}\right)$ with new denture after 5-months follow up when compared to subjects with old denture during maximum voluntary contraction.

\begin{tabular}{|c|c|c|c|c|c|c|c|}
\hline & Mean & SD & Mean & SD & Mean & SD & \\
\hline Right & 231.93 & 59.12 & 163.13 & 42.73 & 68.8 & 40 & $0.000 *$ \\
\hline Left & 230.60 & 46.87 & 171.73 & 75.30 & 58.8 & 61.4 & $0.002 *$ \\
\hline
\end{tabular}

\section{*Significant at $\mathbf{p} \leq \mathbf{0 . 0 5}$}

\section{Discussion:-}

In dentulous subjects, mastication is highly coordinated neuromuscular function that involves fast effective movements of the jaw and continuous modulation of forces in craniofacial system[1]. These muscles have capacity to contract during daily use of the stomatognathic system and maintains the muscle tone in mandibular rest position. Whereas, in edentulous subjects these mechanism acts with marked difference. The loss of natural teeth leads to bone resorption and muscular hypotonicity, which may affect the structures involved in mastication leading to reduced capacity in various functions of stomatognathic system[2]. Masticatory function occurs because of the interrelation between organs of the stomatognathic system, proprioception, brain centers, and occlusal function. Any change in the occlusal characteristics, temporomandibular joint or masticatory muscles may affect the pattern of the masticatory movements and, consequently, their effectiveness. Edentulous persons have reduced capacity to perform various functions of the stomatognathic system such as occlusal forces, tactile thresholds and chewing ability[3].

According to Luis Angeline \& Joseph a. Clayton (1960) A study conducted to evaluate the Quantitative Electromyography of the Masseter Muscle and the usefulness of electromyography depends upon the ability to characterize quantitatively the "normal" population. Moreover, it concluded that, the data now do not permit such a characterization, because of the lack of standardization in recording techniques and the obvious difficulty in expressing the resultant electromyogram in simple quantitative terms[4]. When teeth are lost, a change occurs in the mandibular posture, the speech pattern, esthetics, and deglutition as well as in the individual's social, emotional and psychological behavior. Along with this, some age related changes, such as deterioration in the fast and slow-twitch fibers of the striated muscles, results in impaired muscle force[5]. Age and muscular fatigue affect the function of 
the masticatory system and this is frequently accompanied by tooth loss, prosthetic rehabilitation, and, also by the development of local and systemic circumstances that may greatly influence this physiological process and reduce the masticatory ability of elderly individuals. Because of ageing in the oral cavity, there is a reduction in food intake, as well as opting for softer foods, which leads to an imbalance in nutritional intake. This fact leads to metabolic alterations, which can cause general ageing of tissues of the body[6].

P. Slagter et al (1992) investigated the relationship between the ability of patients with complete dentures to comminute a tough artificial test food, and their answers to questions about chewing experience. It has concluded that the subjective chewing experiences of complete denture wearers were related to ability to comminute test foods and degree of resorption of mandibular ridge was related to comminute test foods[7].The loss of teeth and elimination of periodontal afferent innervations flow lead to changes in the neuromuscular patterns causing the reduced chewing efficiency when new complete denture replaces teeth. Other factors can affect chewing ability in complete denture wearer such as age, gender, personality type, denture experience, denture quality and occlusal schemes. Ellsworth K reported that age did not affect masticatory performance except the patient who is lower than 35 years old and upper than 75 years old. Yamashita $S$ showed that the maximum in masticatory performance found in natural dentition, lower in removable partial denture and the lowest in complete denture wearer[8].Iwao Hayakaw et al (2000) examined changes in masticatory function of complete denture with soft liner and was shown that applying a soft lining material to the mandibular dentures of 6 edentulous patients improved masticatory function with no adverse effect on the muscular tasks[9].

However, the differences were not statistically significant. Restorative procedures, in which the vertical dimension is increased, may influence the mechanisms of chewing cycle. Furthermore, changes in masticatory muscle length resulting from vertical opening may influence the length-tension relationship. An optimum musclular force measured under static conditions occurred at a jaw openings varying between 5 and 10 mm were measured at the first molar region. Hence, increasing the vertical dimension of occlusion may increase bite force during mastication. Thus, an increase in the vertical dimension may influence the masticatory performance both in a negative way, (hampered chewing cycle) and in a positive way (increased bite force and thus better breakage of food particles)[10]. For the patient who has previously worn dentures, the clinician must determine the OVD based on the clinical presentation. By establishing, an appropriate OVD in the patient who has worn the same dentures for 10 years or longer can be challenging. Excessive wear of the denture teeth in a long-term wearer can cause the patient to function at a reduced OVD, possibly compromising the oral craniofacial system.

In addition, older denture wearers may suffer age-related morphologic changes, such as decreased facial height, because of mandibular ridge resorption and a downward and forward rotation of the mandible. These morphologic changes, manifested primarily in the mandibular arch, result in a progressive forward posturing of the mandibular denture that can lead to a reduced horizontal overlap[11].Peyron et al. found a progressive muscular loss as age advances, ranging around $40 \%$ when individuals were 75 years old, and, also a decrease in masticatory performance. The Effect of gender on masticatory performance did not differ in complete denture wearer. On the contrary, gender affected masticatory performance in natural dentition. However, the improvement in denture quality significantly affected masticatory performance in complete denture wearer. Changes in the occlusal vertical dimension are a common procedure in prosthodontic treatment. An increase in the vertical dimension may lead to changes in the orofacial structures, i.e. jaw elevator muscles and temporomandibular joints. Furthermore, an increase in the vertical dimension will lead to a decreased activity of the jaw elevator muscles in postural position. Restorative procedures, in which the vertical dimension is increased, may alter the length of the main jaw elevator muscles as well as the position of the mandibular head in the fossa temporalis[12].

Although, various techniques are available for examining the stomatognathic system, the electromyographic recording is one of the convenient and useful methods because it directly measures muscle activity. Electromyographic techniques have permitted more precise assessment of the muscle functions compared to previously possible clinical observation. Technological development has led to an optimized application of computerized diagnostic systems such as electrognathography[3]. The first documented experiments dealing with EMG started with Francesco Redi's works in 1966. Redi discovered a highly specialized muscle of electric ray fish generated electricity. Marey made the first actual recording of this electrical activity in 1890, who also introduced the term EMG. In 1922, Gasser and Erlanger used an oscilloscope to show the electrical signals from muscles. Clinical use of SURFACE EMG [SEMG] for the treatment of more specific disorders began in 1960's and was used 
by Hardyek The first effort to apply electromyography in dentistry was made by Robert E. Moyers. It used to corroborate the neuropsychological analysis of the factors linked to prosthetic rehabilitation procedures[4].

Electromyography can be used in many clinical and biomedical applications. It acts as a diagnostic tool for identifying neuromuscular diseases, disorders of motor control and, used as a control signal for prosthetic devices such as prosthetic hands, and lower limbs. Electromyographic data representing muscle activity during movements of the mandible (e.g., chewing) are the result of several variables which are operating simultaneously-i.e., continual variation in muscle length, bite dimension, and temporomandibular joint axis. The study of such data has contributed to our knowledge of the relative function of the masticatory muscles and has certainly indicated the potential value of electromyographic data in dentistry.

\section{Various applications of EMG in dentistry:-}

a) It provides an objective means by monitoring changes in muscle activity. b)To study action potentials in actively contracting lingual and masticatory muscles. c)It is used in the treatment of Myofacial Pain Dysfunction where the procedure is called a Auditory or Visual electromyographic feedback, It supplies information to the patient concerning to the muscle activity. d)To study biomechanics of jaw and facial muscle functions. e)EMG is useful for identifying asymmetry of muscle action and particularly for judging the results of the therapy. f)Used for monitoring of nocturnal bruxism and jaw muscle tracking. g)Used as one part of a full assessment protocol to determine if a patient has significant muscle asymmetries possible postural disturbances and significant muscle fatigue.

The usefulness of electromyography depends upon the ability to characterize only quantitatively the "normal" population. At present, data does not permit a characterization based on quality of a muscle activity because of the lack of standardization in recording techniques and the obvious difficulty in expressing the resultant Electromyograms in simple qualitative terms. The two main types of electrodes used for the study of muscle dynamics are surface (or 'skin') electrodes and deep electrodes (usually wire or needle)[13]. Deep electrodes: the electrodes inserted within the muscle by using two thin-coated wire filaments, which introduced by means of a hypodermal needle.They are superior to surface electrodes, as the quality of the electromyogram is better. There are lesser technical artifacts, because distance between the muscle and the electrode remains more constant. There is a risk of infection associated with the use of needle electrodes. They may also be painful. After insertion of the needle, it is always important to confirm that the needle correctly placed by asking the patient to contract the muscle being tested. The most important guideline for adequate needle insertion is to observe and palpate the muscle in contraction, while the test maneuver is being performed. This rule is applicable to almost all superficial muscles. If the needle is correctly positioned this maneuver should easily produce crisp action potentials[5].

The Surface EMG method: most widely and commonly used for recording muscle activity. Surface EMG has been advocated as a "modern scientific approach" because the output from such devices is presumed to be both "quantitative" and "objective". Surface EMG employs surface electrodes, which are non-invasive, painless and reduced risk of infection. Using a surface electrode always presents the possibility of loosening of the electrodes during nerve stimulation. Surface electrodes also give rise to errors when the distance between the electrodes and the muscle changes during muscle contraction. They are much more convenient for clinicians, more acceptable for patients and produce less movement; this is a noninvasive and user-friendly method. Surface electromyogram (EMG) analysis in studies of muscle function has attained increasing attention during recent years and has been applied to assess muscle endurance capacity, anaerobic and lactate thresholds, muscle biomechanics, motor learning, neuromuscular relaxation, muscle soreness, neuromuscular diseases, motor unit activities, and skeletal muscle fatigue[5].

Electromyographic examination of masticatory muscles may have confirming value for clinical diagnosis of myospasm (Spasmodic contraction of a muscle), which characteristically shows a marked increased in EMG activity. Surface EMG of muscles of mastication used routinely as a part of diagnosis and treatment of TMD. The duration of temporary pause in ongoing EMG activity of jaw closing muscles during maximal clench has been found to be longer, on average in a group of TMD patients. Several studies have quantitatively investigated the EMG during postural activity of the mandible and during maximal bite in the intercuspal position. The EMG values for the temporal and masseter muscles have been quantitatively investigated in these studies for control subjects without functional disorders and for patients with functional disorders. 


\section{The purposed usefulness of surface electromyography:-}

A clinical use of SEMG has been proposed for the diagnosis and treatment of TMD. It's based on the assumption that various pathological or dysfunctional conditions can be discerned from SEMG recordings of masticatory muscle activity. The muscle activity including postural hyperactivity, abnormal occlusal positions, functional hyperactivity and hypoactivity, muscle spasms, fatigue and muscle imbalance. SEMG activity has been suggested to be useful in documenting changes in muscle function before and after therapeutic interventions as evidence of successful treatment. SEMG also has been used in biofeedback concerning the awareness and control of nocturnal and diurnal para-functional habits[14].

Masticatory forces in complete edentulous mouth directly depends on- a) the size of muscle creating the forces, b)their position in the mandible, c)type of chewing, d)shape of edentulous alveolar ridges and e)the degree of intermaxillary separation. Bite forces in complete denture wearers are significantly decreased in relation to person with natural teeth. There is fundamental difference in the distribution of functional energy in the complete denture wearer and subjects with intact teeth. Reduction of masticatory efficiency in denture wearers may be caused by irregular activity of masticatory musculature or is the consequences of irregular flow of energy during mastication[3].

\section{Clinical evaluations revealed the following Conditions:-}

Severe decrease in lower face height yielding poor facial esthetics, inadequate fit of complete dentures, worn denture teeth, clinically perceptible deficiency in OVD, acquired protrusive maxillomandibular relationships secondary to resorption, or angular cheilitis. The new complete dentures were made according to the procedure recommended by Zarb et al[12].

Although numerous studies have described comparison of different parameters in edentulous subjects and natural dentition, little comparative information has been regarding the complete denture patients. The data engraved from this study (Table-1) showed significant increase in muscle efficiency of right Anterior Temporalis muscle when compared to left Anterior Temporalis muscle. The mean difference between the values of electrical activity of muscles analysed in maximum voluntary contraction were higher for old denture wearers in comparison with those patients with new complete denture wearers following 5-months follow up. The data obtained from this study (Table-2) showed significant increase in muscle efficiency of right Masseter muscle when compared to left Masseter muscle. The mean difference between the values of electrical activity of muscles analysed in maximum voluntary contraction were higher for old denture wearers in comparison with those patients with new complete denture wearers following 5-months follow up.

According to Seung-Ho Lee Dang-Wan Kang (2002) showed during maximum intercuspation, chewing position, the proportionality of anterior temporalis muscle to masseter muscle activity was lower than other positions, and Naeije Shi found similar results. He also concluded that the temporalis muscle activity is especially sensitive to immediate changes in lateral positioning and that the masseter activity remains unchanged when conditions of stable bilateral occlusal contacts are present. Clinically, the use of new complete denture will allow new occlusal contacts to be reestablished, functionally benefiting the masticatory system by allowing a return to normal parameters.

Zuccolotto et al (2007) showed patients who have been edentulous for over 10 years and have adapted to their unsatisfactory prosthesis may need extra time to reestablish their musculature after changing the prosthesis and it may be necessary to consider new parameter for these patients. The above-mentioned observations indicate that the subjects with old denture wearers show greater muscle activity and hence improved muscle efficiency. The new dentures showed improved facial esthetics, adequate fit, correct maxillomandibular relationships, and anatomical teeth with cuspal morphology[14].

Having new denture after 5-months follow up for adaptability of dentures to the musculature infers minimal muscle activity and greater muscle efficiency. Due to the poor condition of dentures, these edentulous patients follow a diet based on soft foods. For that reason, it was assumed that the musculature would show hypotonicity. By suppressing proprioceptive mechanisms corresponding to the preservation of hypoactive muscle activities and eliminating possible interference from the occlusal relationship of the old dentures, new denture with improved occlusal surface and vertical dimension could promote musculature reconditioning. This fact probably occurred and allowed hypoactive muscles to optimize their electric activity. 
The increase in mean temporalis muscle values is clear evidence of its role in mandibular positioning to establish a new horizontal maxillomandibular relationship. Factors such as OVD, occlusal plane conditioning, integrity and adaptation of old dentures, and the possibility of muscle hypoactivity before treatment, could cause functional imbalances. After being corrected and using new set of complete denture, these were probably characterized by an increase in muscle efficiency. In this study, patients who were evaluated during the whole period of the study, reported that they felt greater comfort and that they were pleased with the aesthetics. Seventy per cent reported that they could chew better when wearing the new set of complete dentures than they did with the old dentures. From these results, it could be concluded that new set of complete dentures allow for neuromuscular reprogramming, which contributes to muscular balance of the masticatory system.

As might be expected the data suggest that there are differences in the levels of muscular activation in elderly individuals during masticatory function. However, there is a need for further long-term studies concerning elderly individuals, with the purpose of elucidating if these results are important for dynamic, specific or non-specific functional activities. By use of new complete denture with established occlusal contacts and vertical dimension, that functionally benefits the masticatory system by allowing a return to normal parameters. Thus, the musculature's EMG activity (anterior temporalis) may be reduced, allowing muscles to work more efficiently during 'tooth' contact. Other authors have affirmed that patients with new dentures increased masticatory efficiency, even with poor muscular adaptation and reduced electrical activity in the masticatory muscles. This increased masticatory efficiency may have developed from muscular adaptation, reestablishment of OVD and occlusal surfaces. The new dentures with correctly positioned cusps facilitated intercuspation and perhaps required a lower amount of force to chew the food. This could lead to greater chewing of food and a better quality diet.

In the present study, the reduction in the number of cycles occurred mainly at the end of mastication. By electromyography, we can also see muscular changes in patients requiring prosthetic rehabilitation; therefore, we can state that rehabilitation need interferes on muscle harmony thus improvement in the efficiency of muscle activity. It is essential to explain to complete denture users, the importance of attending periodical visits in order to evaluate their dentures and oral conditions. These factors are factors intimately linked to aesthetics and functionality.

\section{Limitations of the study:-}

(1)The principal limitation of the present study is the number of analysed subjects, that was under the proper sample size to avoid type II errors. (2)In this study, 5 months was not enough time to observe increased efficiency with the new complete dentures. (3)The complex neuromuscular skills required to overcome the limitations of dentures. Adaptation of neuromuscular system takes an extended time and may be a determinant factor in influencing the EMG activity and this aspect can change the results. (4)Reestablishment of the artificial tooth cusps with anatomic teeth and conventional balanced occlusionpositive outcomes can be expected. (5)Patients who have been edentulous for over 3-5 years and have adapted to their old (unsatisfactory) prosthesis may need extra time to re-establish their musculature after changing the prosthesis and it may be necessary to consider new parameters for these patients. (6)The patients investigated in the present study were healthy physiologically and psychologically and, results may be different in psychologically distressed patients. (7)Electrodiagnostic resources are still far from a concrete professional reality due to the lack of knowledge about the technique along with high-cost equipment.

\section{Further scope of the study:-}

(i)A complementary approach in TMD diagnosis by EMG with other methods is sufficient if applied. (ii)Improved masticatory efficiency would be explained by enhanced bilateral balanced occlusion obtained with the new dentures. (iii)The behavior of the dynamics masticatory muscle activity, like chewing swallowing and in rest postural position underlines the importance of an integrated analysis of both kinematics and EMG activity in the follow up of patients with new dentures. (iv)The studies should clarify the association among masticatory efficiency and alterations in anatomical occlusal form. (v)Further investigations are needed to explore the relationship between occlusal features and muscular activity, designed following specific criteria (randomization, inclusion and exclusion criteria, similarity between groups at baseline, detailed description of the protocols to facilitate replication, blinding methods) in order to establish if a causal association between these variables really exist, thus avoiding spurious associations. (vi)Longitudinal studies with the purpose to appraise the long-lasting effects of occlusal disturbance on the activity of masticatory muscles. (vii)Finally, a greater accuracy of the electromyography would be desirable to confer to the results obtained an absolute reliability. 


\section{Conclusion:-}

According to the methodology used and according to the results obtained it may be concluded that the electrical activity of Anterior Temporalis muscle and Masseter muscle during maximum voluntary contraction was reduced; hence, muscle efficiency has been improved after 5 months with the new complete denture in place. The difference was statically significant. The results show that new dentures or improvements in occlusion and vertical dimension produce a positive benefit to the patient by reducing the muscle effort during chewing without affecting masticatory performances. And it also indicate that in edentulous subjects with a old denture used for several years, after the delivery of a new denture 5 months of follow-up, the EMG activity of right side of the anterior Temporalis muscle decreases hence, the muscle efficiency is improved as compared to other group of muscles examined. The reduction in muscle effort is likely to cause less tissue trauma and in the end may minimize residual ridge resorption.

A new complete denture allows for neuromuscular reprogramming, which contributes to muscular balance of the masticatory system. With wear of old complete dentures over a period (more than 5-6 years), lead to the compromise in esthetic and function with reduced masticatory efficiency. Thus, recognition of these expected gains to the patient is important for the dentist to make treatment recommendations about making new dentures for every 3-5years. Moreover, improvement in occlusal surface (anatomic cusp), reestablishment of new vertical dimensions significantly improves the esthetic, function and increased masticatory efficiency. However, further improvement in muscle efficiency in edentulous patient leading to better nutrition and digestion. All these factors considered for physical and psychological benefit to the patient's health, condition and well-being.

\section{Acknowledgment:-}

A special note of thanks to Dr. Muddapur for helping me out in every step of my data entry, analysis and understanding of the statistical procedures employed and Dr.Datta Nadiger MD, DNB Neurosurgeon for all the technical help and support involved in this study.

\section{References:-}

1. Marcelo Coelho Goiato et al (2007):Electromyographic evaluation of Masseter and anterior Temporalis muscles in resting position and during maximum tooth clenching of edentulous patients before and after new complete dentures. Acta odontol. Latinoam; 20 (2): 67-72.

2. Marcelo Coelho Goiato et al (2010): Analysis of Masticatory Cycle Efficiency in Complete Denture Wearers. J Prosthet Dent; 19: 10-13.

3. Haifa Ashraf(2011). To determine the influence of complete denture prosthesis on masticatory muscle activity in elderly patients-an invivo study. Int J Prosthet Rest Dent; 1(1): 35-40.

4. Juliana malta et al ( 2006): Electromyography applied to chewing muscles. Acta Ortop Bras.; 14(2): 106-107.

5. Henrique Casselli et al (2007): Electromyographic evaluation of rehabilited edentulous patients. Braz Oral Res.; 21(4): 355-61.

6. Gunne HS et al (1985): The effect of new complete dentures on mastication and dietary intake. Acta Odonto Scand.; 43: 257-268.

7. Iva Alajbeg Melita et al (2003): Electromyographic evaluation of masticatory muscle activity in patients with temporomandibular dysfunction. Acta Stomat Croat.; 37(2): 141-145.

8. Luis Angelone et al(1960): An approach to quantitative electromyography of the Masseter muscle. J D Res; 39(1): 17-23.

9. Zarb GA (1982). Oral motor patterns and their relation to oral prostheses. J Prosthet Dent; 47:472-478.

10. P. A. Pro" schel et al (2010):Indications for jaw gape-related control of relative muscle Activation in sequent chewing strokes. Journal of oral rehabilitation 37; 178-184.

11. Iwao Hayakawa et al (2000): Changes in masticatory function of complete denture wearers after relining the mandibular denture with a soft denture liner. Int J Prosthodont; 13: 227-231.

12. Maria Teresa Botti Rodrigues dos Santos et al (2008): Electromyographic evaluation of anterior Temporalis and Masseter muscles in patients with cerebral palsy before and after postural stabilization. Journal of Disability and Oral Health; 9(2): 59-62.

13. Sven E. Widmalm et al (2007): Clinical Use of Qualitative Electromyography in the Evaluation of Jaw Muscle Function: A Practitioner's Guide. The journal of craniomandibular practice.; vol. 25 (1): 63-66 .

14. H c. Karkazis et al (1998): Surface EMG activity of the Masseter muscle in denture wearers during chewing of hard and soft food. J Oral Rehabil; 25: 8-4. 
15. Kawazoe Y et al (1979): Relation between integrated electromyographic activity and biting force during voluntary isometric contraction in human masticatory muscles. J Dent Res; 58:1440-1449.

16. Tallgren A et al (1986): Changes in jaw relations and activity of masticatory muscles in patients with immediate complete upper dentures. J Oral Rehabil; 13:311-324.

17. Goiato MC et al (2008): Electromyographic activity of the mandible muscle s at the beginning and end of masticatory cycles in patients with complete dentures. Gerodontology; 54: 138-143.

18. Peter A. Proschel et al (2001): Preconditions for estimation of masticatory forces from dynamic EMG and isometric bite force-Activity relation of elevator muscles. Int J Prosthodont; 14: 563-569.

19. Gunne HS et al: Masticatory efficiency of complete denture patients. A clinical examination of potential changes at the transition from old to new denture Acta Odontol Scand. 1982; 40(5): 289-97.

20. DcSven E. Widmalm et al (2007): Clinical Use of Qualitative Electromyography in the Evaluation of Jaw Muscle Function: A Practitioner's Guide. The journal of craniomandibular practice.; 25(1): 63-73.

21. Ignacio Ardizone et al (2010): Electromyographic study of activity of the Masseter and anterior Temporalis muscles in patients with temporomandibular joint (TMJ) dysfunction- Comparison with the clinical dysfunction index. Med Oral Patol Oral Cir Bucal.; 15 (1): 14-19

22. Sakar O et al (2011): Reliability and comparison of two facial measurements to detect changes of occlusal vertical dimension in complete denture wearers. Gerodontology.; 28(3): 205-8.

23. Keith A et al (2003): Reestablishing occlusal vertical dimension using a diagnostic treatment prosthesis in the edentulous patient: a clinical report. J Prosthodont.; 12(1): 30-6.

24. L. W. Olthoff et al:. Influence of occlusal vertical dimension on the masticatory performance during chewing with maxillary splints Journal of Oral Rehabilitation 2007 34; 560-565

25. Olcay S et al (2011): Reliability and comparison of two facial measurements to detect changes of occlusal vertical dimension in complete denture wearers Gerodontology; 28: 205-208

26. Jemt T ( 1981). Chewing patterns in dentate and complete denture wearers - recorded by light-emitting diodes. Swed Dent J; 5:199-205.

27. Fujimori T et al (2002): Effects of a denture adhesive on mastication functions for complete denture wearers. $\mathbf{J}$ Med Dent Sci; 49:151-156.

28. Agerberg G (1988). Mandibular function and dysfunction in complete denture wearers-a literature review. J Oral Rehabil; 15:237-249.

29. Gervais RO et al (1989): Masseter and Temporalis eletromyographic activity in asymptomatic, subclinical and temporomandibular joint dysfunction patients. Cranio; 7:52-57.

30. Hagberg C (1986). The amplitude distribution of electromyographic activity of masticatory muscles during unilateral chewing. J Oral Rehabil; 13:567-574. 